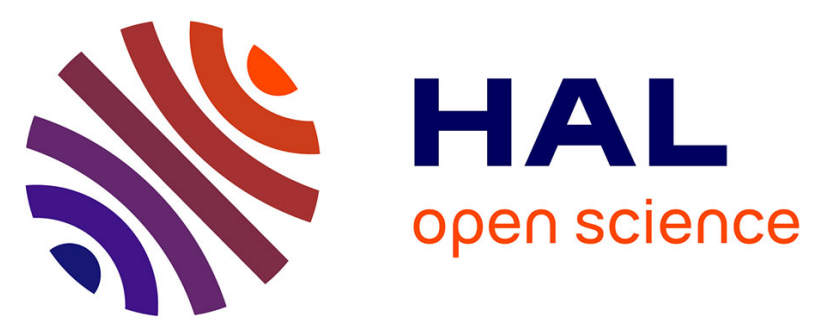

\title{
Experimental evidence of nanometer-scale confinement of plasmonic eigenmodes responsible for hot spots in random metallic films
}

Arthur Losquin, Sophie Camelio, David Rossouw, Mondher Besbes, Frédéric Pailloux, David Babonneau, Gianluigi A. Botton, Jean-Jacques Greffet, Odile Stéphan, Mathieu Kociak

\section{To cite this version:}

Arthur Losquin, Sophie Camelio, David Rossouw, Mondher Besbes, Frédéric Pailloux, et al.. Experimental evidence of nanometer-scale confinement of plasmonic eigenmodes responsible for hot spots in random metallic films. Physical Review B: Condensed Matter and Materials Physics (1998-2015), 2013, 88 (11), pp.115427. 10.1103/PhysRevB.88.115427 . hal-01335075

\section{HAL Id: hal-01335075 \\ https://hal-iogs.archives-ouvertes.fr/hal-01335075}

Submitted on 21 Jun 2016

HAL is a multi-disciplinary open access archive for the deposit and dissemination of scientific research documents, whether they are published or not. The documents may come from teaching and research institutions in France or abroad, or from public or private research centers.
L'archive ouverte pluridisciplinaire HAL, est destinée au dépôt et à la diffusion de documents scientifiques de niveau recherche, publiés ou non, émanant des établissements d'enseignement et de recherche français ou étrangers, des laboratoires publics ou privés. 


\title{
Experimental evidence of nanometer-scale confinement of plasmonic eigenmodes responsible for hot spots in random metallic films
}

\author{
Arthur Losquin, ${ }^{1}$ Sophie Camelio, ${ }^{2}$ David Rossouw,${ }^{3}$ Mondher Besbes, ${ }^{4}$ Frédéric Pailloux,${ }^{2}$ David Babonneau, ${ }^{2}$ \\ Gianluigi A. Botton, ${ }^{3}$ Jean-Jacques Greffet, ${ }^{4}$ Odile Stéphan, ${ }^{1}$ and Mathieu Kociak ${ }^{1, *}$ \\ ${ }^{1}$ Laboratoire de Physique des Solides CNRS/UMR8502, Université Paris-Sud, Orsay, 91405, France \\ ${ }^{2}$ Institut P', Département Physique et Mécanique des Matériaux, UPR 3346 CNRS, Université de Poitiers, 86962 \\ Futuroscope Chasseneuil, France \\ ${ }^{3}$ Department of Materials Science and Engineering, McMaster University, Hamilton, Canada \\ ${ }^{4}$ Laboratoire Charles Fabry, Institut d'Optique, Université Paris-Sud, CNRS, Palaiseau, 91127 France \\ (Received 23 May 2013; revised manuscript received 28 August 2013; published 23 September 2013)
}

\begin{abstract}
We report on the identification and nanometer scale characterization over a large energy range of random, disorder-driven, surface plasmons in silver semicontinuous films embedded in silicon nitride. By performing spatially resolved electron energy loss spectroscopy experiments, we experimentally demonstrate that these plasmons eigenmodes arise when the films become fractal, leading to the emergence of strong electrical fields ("hot spots") localized over few nanometers. We show that disorder-driven surface plasmons strongly depart from those usually found in nanoparticles, being strongly confined and randomly and densely distributed in space and energy. Beyond that, we show that they have no obvious relation with the local morphology of the films, in stark contrast with surface plasmon eigenmodes of nanoparticles.
\end{abstract}

DOI: 10.1103/PhysRevB.88.115427

PACS number(s): 78.66.Bz, 71.55.Jv, 73.20.Mf, 78.20.-e

\section{INTRODUCTION}

Disorder affects the physical properties of materials in various ways. However, one of the most striking and universal effects of disorder is the appearance of localization effects. For example, the propagation of wave or particles in a medium can be subtly affected by a certain amount of disorder. A famous example is the weak localization (or coherent backscattering) phenomenon appearing for electrons (or photons), resulting in a reduced conductivity (transmittance) with respect to the classical formulas, and which is a signature of coherence preservation along closed paths. ${ }^{1}$ As the disorder increases, weak localization tends to the Anderson localization, a drastic and entirely disorder-driven change from a conducting to an insulating state.

In an alternative description, disorder can radically affect the eigenmode structure of the material of interest. The most striking example is the prediction in the early nineties of the appearance of specific optical eigenmodes in disordered fractal optical media. ${ }^{2}$ Such modes were later predicted to exhibit exceptional properties. ${ }^{3}$ Indeed, they were assumed to happen over a broad range of wavelengths in the infrared (IR), and to have intense, extremely localized (over few nanometers), and widely distributed electric fields. They were also supposed to be a natural source of field enhancement for surface enhanced Raman scattering (SERS). ${ }^{4}$ Surface plasmon (SP) eigenmodes with similar properties were later predicted for semicontinuous metal films around the percolation threshold, ${ }^{5,6}$ which are well known fractal systems. ${ }^{7}$ These eigenmodes gave a natural explanation for previously measured abnormal absorption properties $^{8}$ as well as calculated light induced electric fields ${ }^{9}$ in percolated films. Again, these calculated electric fields appeared to be intense, localized and randomly distributed in space in a broad spectral range, and were commonly referred to as hot spots (HS). ${ }^{9}$

These surprising properties are totally different from what happens in simple metallic systems like single nanoparticles, for which the SP eigenmodes can be easily deduced from the nanoparticles shape and size. ${ }^{10}$ This triggered an intense experimental effort towards the observation of the SP eigenmodes of percolating metal films. Such samples were observed with scanning near-field optical microscopy (SNOM). ${ }^{11}$ The maps obtained displayed localized strong intensity maxima. Despite a limited spatial resolution that did not allow retrieving their nanometer scale spatial structure and a restricted spectral range of excitation insufficient to demonstrate their broadband character, these maxima were interpreted as to be the HS. After this early pioneering work, similar measurements have been extended to several excitation wavelengths and metallic regimes using various near-field techniques. ${ }^{12-17}$ The observation of local spots of very high intensity was confirmed. However, the lack of spatial resolution, clearly pointed out in recent works, ${ }^{15,17}$ as well as a monochromatic excitation, prevented a proper description of the spectral and spatial properties of individual eigenmodes. In particular, the predicted high spatial localization of such SP eigenmodes could not be proven.

In this paper, we report the observation of the nanometer spatial structure of random eigenmodes arising over a broad spectral range in semicontinuous metal films around the percolation treshold. By correlating at the nanometer scale the intensity of their associated HS, their spectral signature and the structure of the medium, we provide the experimental evidence of the predicted modal structure, high localization and random character of the eigenmodes of disordered media. We also show that the properties of the eigenmodes have no obvious relationship to the local structure of the underlying medium.

\section{METHODS}

We have used spatially resolved electron energy loss (EEL) spectroscopy in a scanning transmission electron microscope (STEM-EELS). This technique has been proven essential to 
map with unique spatial resolution SP eigenmodes of highly symmetric metallic nano-objects, ${ }^{18,19}$ or of more complex nanoparticles and nanoporous films. ${ }^{20,21}$ Using STEM-EELS allowed us to acquire local spectra at the nanometer scale with no inhomogeneous broadening. Therefore the observed spectral resolution takes advantage of our very high spatial resolution. Since a single local spectrum consists of only a few peaks, we can separate these peaks spectrally so that their complete characteristics can be recovered. We also point out that, contrary to many near-field optical setups, ${ }^{22}$ the STEMEELS probe is noninvasive. To interpret our experiments, we have used the concept of electromagnetic local density of states (EMLDOS), ${ }^{23}$ to which the quantity measured in STEM-EELS is very close $\mathrm{e}^{24,25}$ and which has been extensively used to understand the near-field optical properties of semicontinuous films. ${ }^{26-28}$ Indeed, the spatial variations of the EMLDOS at the eigenfrequency of a given SP eigenmode reflect the electric field intensity local variations of the eigenmode. ${ }^{25}$

\section{A. Sample preparation}

The samples consist of silver sandwiched between two amorphous $\mathrm{Si}_{3} \mathrm{~N}_{4}$ films. The preparation was carried out by alternate ion beam sputtering (IBS) in a NordikoTM 3000 vacuum chamber and has been described in detail in Ref. 29. A thick $\mathrm{Si}_{3} \mathrm{~N}_{4}$ film was first deposited at $200^{\circ} \mathrm{C}$ onto a faceted $\mathrm{NaCl}$ single crystal and was then sputtered at room temperature with $1 \mathrm{keV} \mathrm{Xe}^{+}$ions at a sputtering angle of $55^{\circ}$ with respect to the normal of the mean (001) surface and with a total fluence of $5.4 \times 10^{16}$ ions $/ \mathrm{cm}^{2}$. It is well known that IBS under off-normal incidence may result in the generation of unidirectional ripples. The phenomenon, which is related to the interplay between surface roughening (due to the curvature dependence of the sputtering yield) and the smoothing of the surface by different relaxation mechanisms, strongly depends on the sputtering conditions (angle of incidence, temperature, ion energy and mass, total fluence, etc.). Therefore only the (001) facets of the $\mathrm{NaCl}$ surface exposed to the defocused $\mathrm{Xe}^{+}$flux at an incidence of $55^{\circ}$ develop nanoripples (oriented perpendicular to the ion beam and with a period around $25 \mathrm{~nm}$ ), while other areas with different orientations remain flat. The inhomogeneously prepatterned surface of the $\mathrm{Si}_{3} \mathrm{~N}_{4}$ buffer-layer was then used as template for the subsequent growth of silver occurring by the Volmer-Weber mechanism. Silver deposition was accomplished at room temperature with a glancing angle of $5^{\circ}$ from the mean surface and with the metallic flux being oriented perpendicular to the periodic ripples. Shadowing effects associated with a preferential diffusion of silver atoms along the ripples resulted in the production of aligned ellipsoidal nanoparticles in the rippled areas, ${ }^{29}$ while tortuous or percolatinglike structures were formed in the flat areas where the deposition rate was higher. Finally, after deposition of an additional $\mathrm{Si}_{3} \mathrm{~N}_{4}$ capping-layer at $200{ }^{\circ} \mathrm{C}$, the sample was floated in purified water and the resulting freestanding thin film was collected on microscope copper grids.

\section{B. STEM EELS acquisition and processing}

During a measurement, a nanometric electron probe is scanned over the sample. Both the local topography of the
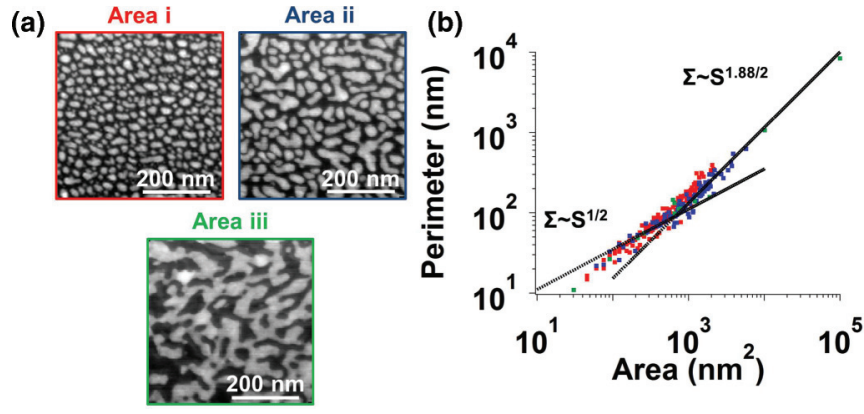

FIG. 1. (Color online) (a) HAADF images of areas i to iii, where silver appears bright. (b) Log-log plot of the perimeters of the metal clusters found in each area as a function of their surfaces. Red, blue, and green dots relate, respectively, to the areas i, ii, and iii. For small clusters, the perimeter roughly scales with the square root of the surface, which is characteristic of Euclidian shapes. The slope increases for large clusters, as expected for fractal shapes.

material [high angle annular dark field (HAADF) signal] and an EEL spectrum are recorded at each probe position simultaneously. After a complete scan, a HAADF image of the area, in which silver appears bright over a dark background, is obtained, and can be compared to a full spectroscopic data set.

Most of the STEM-EELS measurements were performed with a vacuum generators (VG) HB-501 STEM equipped with a cold field emission electron gun and operated at $100 \mathrm{kV}$ (data in Figs. 1-4). ${ }^{18,20}$ Additional data (see Fig. 5) were obtained with a FEI Titan 80-300 kV Cs-corrected TEM equipped with a monochromator and working in STEM operation mode at $80 \mathrm{kV} .{ }^{19}$ In both types of experiments, the sample was cooled down by liquid nitrogen. Convergence and collection angles were respectively set to 7 and $11 \mathrm{mrad}$ for data acquired with the VG STEM, and to 7 and $14 \mathrm{mrad}$ for acquisition with the FEI TEM. The spectral resolutions of the data sets are respectively estimated as 200 and $60 \mathrm{meV}$ after the Richardson-Lucy deconvolution. ${ }^{30}$

EEL probability ${ }^{24}$ spectra were obtained after normalization of each EEL spectrum and subtraction of the zero loss peak (ZLP). ${ }^{18,20}$ Multipeak fitting was performed by fitting a sum of Gaussian functions and a sigmoidal background within each spectrum of the data set. ${ }^{20}$ Conditions on minimum noise level, number of binomial smoothing operations, and maximum number of peaks were imposed. Maps of amplitude, central energy, and full width at half maximum (FWHM) of the peaks whose central energy is found in a given energy range were obtained by assigning to each probe position the amplitude, central energy, and FWHM of the Gaussian function that was fitted within the corresponding spectrum in this energy range. For all the maps shown in this paper, care was taken that only one fitted Gaussian function has its center in the indicated energy range.

In particular, in order to estimate the spatial extension, eigenenergy, and damping rate of a single eigenmode, the peaks whose central energies were found within very narrow energy windows were selected to provide maps corresponding to this eigenmode. A 2D Gaussian function was fitted on the amplitude map, allowing defining the size of the eigenmode as the FWHM of this Gaussian function. Then, to evaluate its damping rate and eigenenergy, only the pixels of the FWHM 
(a)
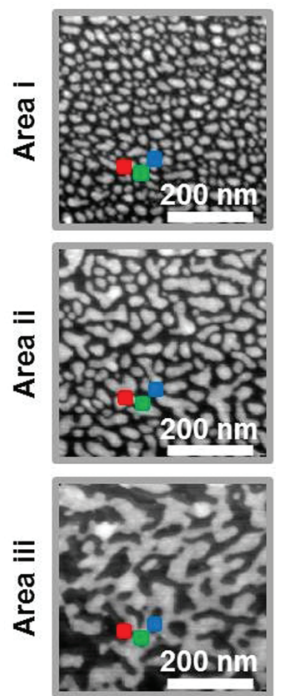

(b)
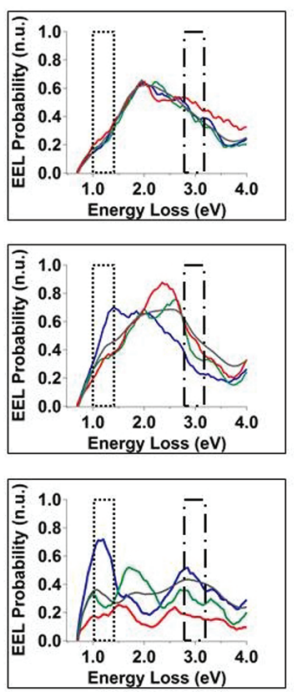

(c)

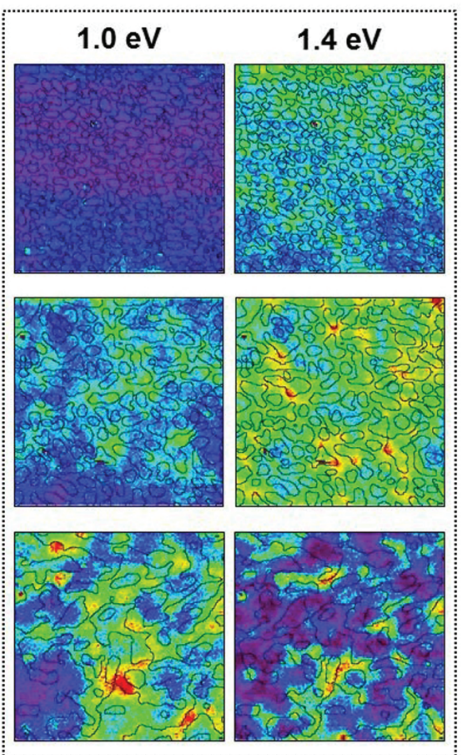

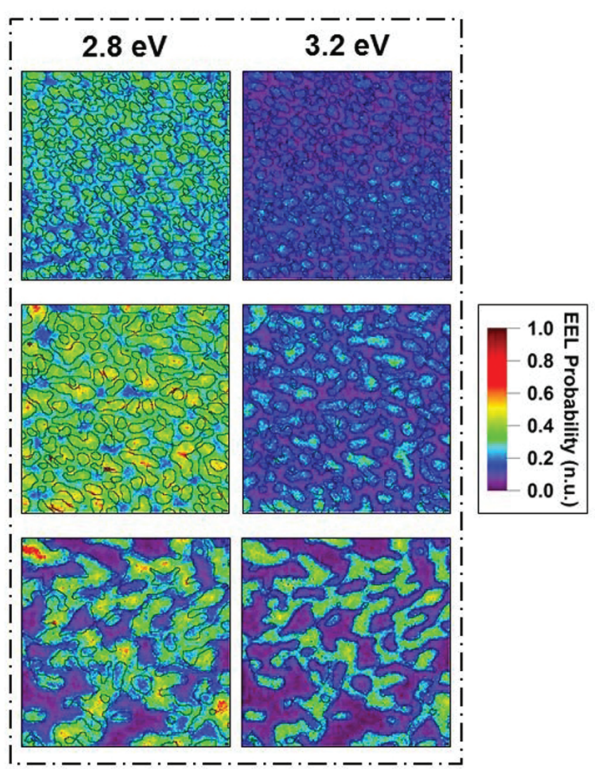

FIG. 2. (Color online) (a) HAADF images of areas i-iii. (b) EEL probability local spectra (colored) corresponding to the probe positions indicated in (a) and mean EEL probability spectra (grey) obtained by averaging the set of spectra acquired in the whole area. The dashed boxes demarcate some low-energy and high-energy regimes. (c) Spatial variations of the EEL probability at the energy loss values of 1.0 and $1.4 \mathrm{eV}$ (low-energy regime) and 2.8 and $3.2 \mathrm{eV}$ (high-energy regime). The superimposed contour line is the sample morphology profile obtained from the HAADF images.

and energy maps that correspond to pixels of the amplitude map whose value is larger than the half maximum of the Gaussian function were kept to remove noise effects and contributions from aberrant fits. The eigenenergy of the mode was thus directly defined as the mean value of the new energy map. For the damping rate, the values of the remaining pixels of the FWHM map were deconvolved to remove the effect of the finite spectral resolution, which induces some peak broadening as described in Ref. 31. Precisely, assuming that the spectrum one actually measures is approximately a sum of Gaussian functions, the FWHM of each physical peak was estimated from the square root of the subtraction of the square of the FWHM of the measured peak and the square of the FWHM of a deconvolved ZLP recorded in vacuum. The damping rate was then defined as half the mean value of the resulting FWHM map. (a)

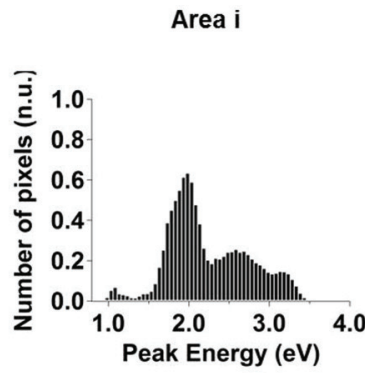

(b)

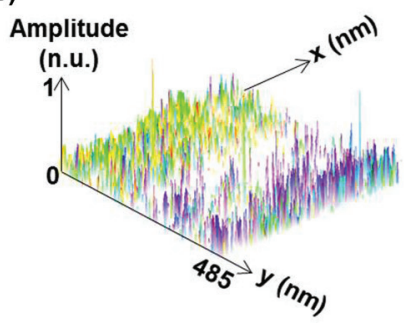

Area ii
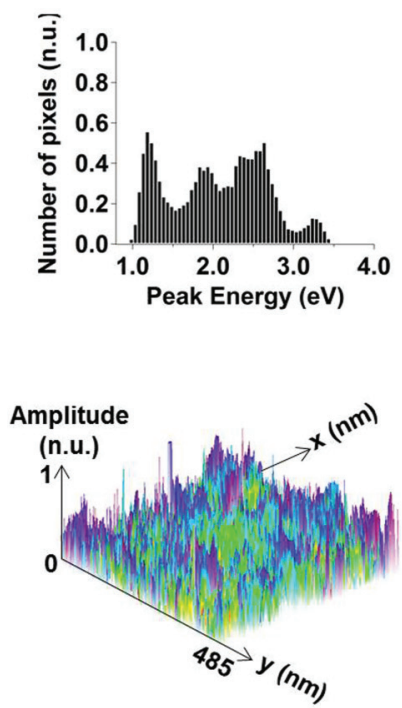

Area iii
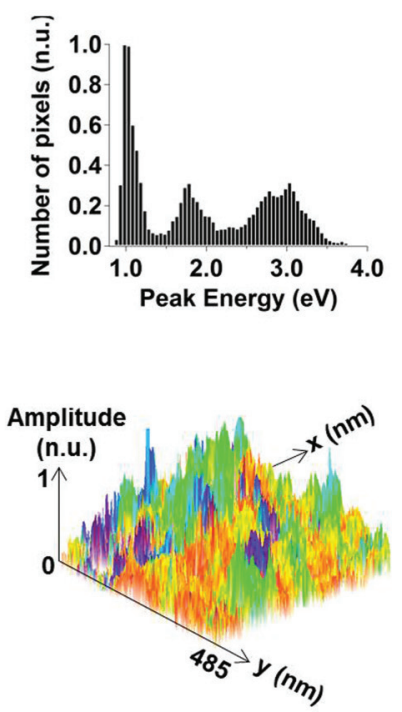

FIG. 3. (Color online) (a) Histograms of the percentage of pixels in areas i to iii in which a peak with a given central energy was found, weighted by the amplitude of the peaks. (b) Map combining the amplitude (in height) and central energy (through a color code) of the peaks whose central energy is between 0.8 and $1.5 \mathrm{eV}$. A pixel is blanked when no peak was found in this spectral range. 
(a)

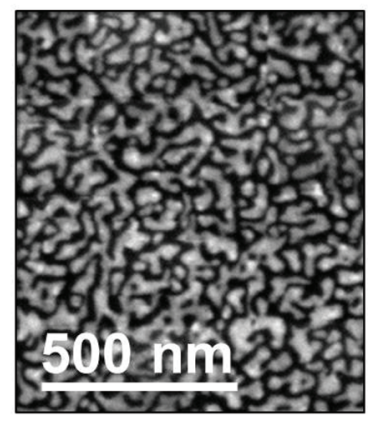

(b)
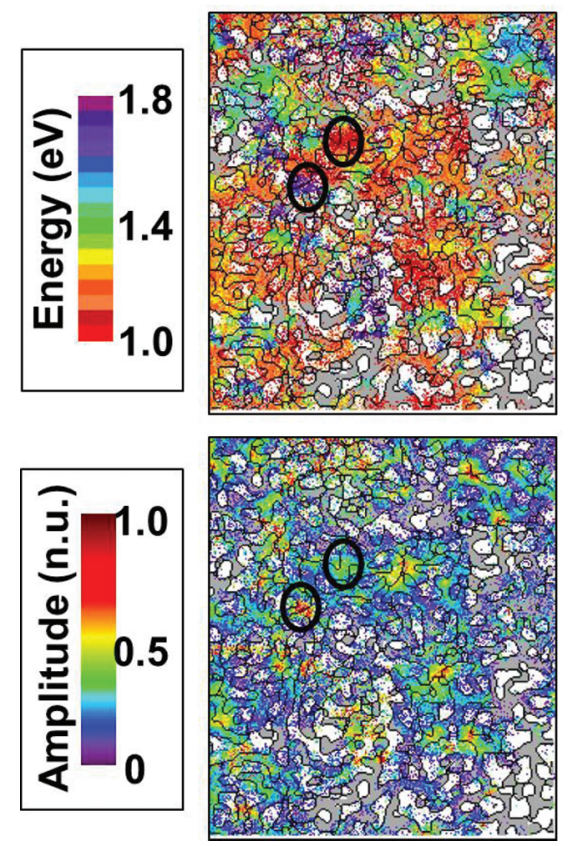

FIG. 4. (Color online) (a) HAADF image of a large semicontinuous area slightly before the percolation threshold. (b) Maps of the energy and amplitude of the peaks whose central energy is between 1.0 and $1.8 \mathrm{eV}$. When no peak is found in these spectral ranges, the pixel is either blanked if the local material is silver, or greyed if the local material is silicon nitride. The superimposed contour line is the sample profile obtained from the HAADF image. The black circles stress two different eigenmodes.

\section{Numerical simulations}

To study numerically a percolating system, the morphology of area iii (see further) was extracted from its HAADF image. An area of size of $400 \mathrm{~nm}^{2}$ was selected. The experiments were modeled by computing the z-projected EMLDOS (zEMLDOS), deduced from the power emitted by a pointlike dipole vertically polarized at a distance of $5 \mathrm{~nm}$ above the film. The frequency of the dipole has been scanned in order to obtain zEMLDOS spectra. The computation was based on the finite element method (FEM) where the electric field is discretized with edge elements. ${ }^{32}$ Perfectly matched layers are used to model boundary conditions. The central energy and FWHM of some zEMLDOS peaks were estimated from the calculated spectra.

\section{RESULTS AND DISCUSSION}

The HAADF images of three areas, representative of an extensive data set, are shown in Fig. 1(a). These areas will be referred to as areas $\mathrm{i}$ to iii. These nanoscale structures are illustrative examples of metal-dielectric composites with various metal filling fractions and geometrical complexities, from aligned and disconnected small nanoparticles (area i) to a percolatinglike, large, and tortuous cluster (area iii).

To classify the morphology of each region among those of semicontinuous films reported in the literature, a geometrical analysis has been performed by thresholding, binarizing, and performing a contour detection of the silver clusters in each image. Figure 1(b) shows the plot in log-log scale of the perimeters of the metal clusters as a function of their areas. In each area, the perimeter of the smallest clusters is found to roughly scale with the square root of the surface, which is typical to objects with Euclidian shapes. In contrast, the largest clusters confirm a power law with an increased exponent reflecting their tortuous geometry. Such an increased slope is characteristic of fractal objects, for which it can be shown that the perimeter scales with the surface as $\Sigma \sim S^{1.88 / 2},{ }^{16}$ a relationship quite well reproduced here. The three areas thus differ from their fractal cluster-Euclidian cluster ratio, which increases from area $\mathrm{i}$ to iii, as expected for semicontinuous metal films with a growing metal coverage. Area i corresponds to a low metal coverage regime, where all the clusters are basically small and Euclidian, while area iii is clearly close to the percolation threshold, where the fractal geometry of the percolating clusters dominates the geometry of the system. This fractal geometry is known to be responsible for the unusual optical properties of semicontinuous films around the percolation threshold. ${ }^{33}$ The set of SP eigenmodes is thus expected to change depending on the investigated area, which we demonstrate properly in the following.

Examples of local EEL probability spectra, obtained from EEL spectra recorded at probe positions separated by $40 \mathrm{~nm}$, are shown in Fig. 2(b). The corresponding probe positions are indicated on the HAADF images shown in Fig. 2(a). As clearly apparent from the data sets, the growing metallic coverage from area $\mathrm{i}$ to iii is accompanied by a progressive spectral complexity. The spectral structure goes from a Mietype spectrum with a peak at around $1.9 \mathrm{eV}$ in area i, to a complex multiplicity of peaks in area iii. Mapping the EEL probability over each area at a given value of the energy loss is done in Fig. 2(c) for four different energy values. The two first values were chosen to be part of the IR region (low-energy regime), while the two others are close or within the ultraviolet (UV) (high-energy regime). From simple inspection of the data, it may be tempting to state that HS, which are traditionally loosely defined as local spots of increased intensity observed at constant excitation wavelength with a near-field optical setup, ${ }^{9,11-15}$ are observed whatever the area and energy. However, the strength of the various spots, within which the EEL probability clearly increases locally, grows from area $\mathrm{i}$ to iii. The main differences between the three areas are found in the low-energy regime, where in area iii changing the energy as little as $400 \mathrm{meV}$ has a strong effect on the shape and spatial arrangement of these spots. Their spatial structures appear complex and show no obvious correlation 
with the local substrate geometry. In this sense, their nature drastically changes as compared to the spots seen in areas $\mathrm{i}$ and ii in the low-energy regime. Yet this is no longer the case in the high-energy regime: the spots are now, irrespective of the area, weakly energy dependent and exclusively located on the metal (as can be deduced from the comparison of the maps and corresponding HAADF images). This set of data clearly points to the fact that these spots can be related to the long predicted HS only at low energy and close to the percolation threshold.

Moreover, these data suggest the emergence of localized SP eigenmodes $5,6,9,11,15,16,34$ at low energies around the percolation threshold. However, we emphasize that interpreting the data in terms of eigenmodes requires further processing. Indeed, as a result of dissipation, the peaks broaden so that the EMLDOS at a given energy is a mix of eigenmodes of different eigenfrequencies. This broadening further increases in our measurements due to the set-up finite spectral resolution. As a consequence, to isolate a single eigenmode, we need to extract locally its complete spectral characteristics. When this is done for each excitation probe position, the spatial structure of the eigenmode can be recovered. Automatic multipeak fitting was thus performed in each spectrum of the three areas. Peaks were successfully isolated. Their central energy, FWHM and amplitude were then attributed to the eigenenergy, damping rate, and local projected electric field intensity of each locally excited SP eigenmode. ${ }^{25}$ The number of probe positions for which a peak with a given central energy was found in the corresponding spectrum, weighted by the peak amplitudes, ${ }^{20}$ is shown by the histograms in Fig. 3(a). As demonstrated by the histograms, while all the areas have similar spectral features at high energies, they mostly differ from the presence of peaks at energies lower than $1.5 \mathrm{eV}$. The number of these peaks progressively increases from area i to iii. In the maps in Fig. 3(b), the amplitude and central energy of these peaks, whose central energy ranges between 0.8 and $1.5 \mathrm{eV}$, are displayed simultaneously, respectively in height and through a color code. These maps are the clear illustration of the gradual appearance of strong peaks spreading continuously towards the IR from area i to iii. Moreover, we can also observe local maxima of increasing strength and confinement within the spatial variations of the peak amplitudes.

We further consider a large semicontinuous area slightly before the percolation threshold, whose HAADF image is shown in Fig. 4(a). Figure 4(b) shows maps of the energy and amplitude of the peaks whose central amplitude is between 1.0 and $1.8 \mathrm{eV}$. As obvious from these maps, each local maximum within the amplitude map is clearly found to be associated to a given constant peak energy within the energy map (see, for instance, the two black circles). We are then able to make a distinction between these amplitude maxima through their associated peak energy. Otherwise speaking, we are able to separate eigenmodes whose projection of the electric field intensities are given by the amplitude map, through their eigenenergy, which is shown by the energy map. We here stress again a key point: as a result of the peak broadening we previously made reference to, a HS can be the result of a mix of different eigenmodes. The description of an eigenmode then requires the knowledge and analysis of the whole spectrum at each point of the medium. We note that a large number of eigenmodes with close yet different eigenenergies coexist not only locally but also over very large scales, which makes such a medium spatially and spectrally dense. Extending this analysis to areas $\mathrm{i}$ to iii, we can thus conclude, from a quick inspection of the maps in Fig. 3(b), that increasing the metal coverage towards the percolation threshold leads to a progressive appearance of multiple eigenmodes of low eigenfrequencies with growing field strength and confinement. These IR eigenmodes thus result from the fractal geometry of the medium, as predicted in Ref. 9. Similarly to the spots visible in the spatial variations of the EEL probability at low energies (Fig. 2(c)), the nanometric areas within which the fields of the eigenmodes are locally confined do not show any obvious correlation with the local substrate geometry. This is a clear signature of the extraordinary, random character of these eigenmodes, which are drastically different from the eigenmodes of nanoparticles.

To characterize these eigenmodes further, we have performed a detailed analysis of a small structural detail of another semicontinuous area, whose HAADF images are shown in Fig. 5(a). Local EEL spectra are displayed in Fig. 5(b). The measurement was performed with an improved spectral resolution, thus allowing the detection of intense peaks down to around $0.5 \mathrm{eV}$. Maps of the amplitude, central energy, and FWHM of the peaks whose central energies are part of two narrow spectral ranges are shown in Fig. 5(c). These spectral ranges were chosen to select two different eigenmodes. Each eigenmode is characterized spatially by a well defined amplitude maximum, position over the substrate, and spatial extension; also spectrally, by a well defined eigenenergy and damping rate. Their spatial extension, eigenenergy, and damping rate are estimated as detailed in the Methods section. Precisely, their spatial extents are found to be $8 \times 5$ and $8 \times 8$ $\mathrm{nm}^{2}$, and their eigenenergy and damping rate are, respectively, evaluated as 1.31 and $1.61 \mathrm{eV}$ and 43 and $48 \mathrm{meV}$.

To obtain more quantitative insight, further analysis was performed on a few additional eigenmodes. Figures 5(d) and 5(e) (red dots) show, respectively, their spatial extent and damping rate as a function of their eigenfrequency. Figure 5(d) confirms that many of the eigenmodes have true nanometric dimensions, which make them differ further from classical SP eigenmodes of nanoparticles. We note that a few of them spread over space more than the others. On the other hand, Fig. 5(e) shows that the damping rates of the eigenmodes are relatively high, similarly to the SP eigenmodes of single nanoparticles. ${ }^{31}$ To corroborate these findings, which seem to disagree with previously published studies suggesting SP resonances with FWHM of 20 to $40 \mathrm{meV},{ }^{11,15}$ we also studied numerically a percolating system by computing the projection of the EMLDOS along the axis of the electron. When taking the dielectric properties from Ref. 35, the resonances within an EMLDOS spectrum were found too narrow to satisfyingly account for the experimental results. Nevertheless, it is known that for thin films, the imaginary part of the dielectric constant increases as the films thickness decreases. ${ }^{36}$ We have found a qualitative agreement by using an imaginary part of the dielectric constant multiplied by a factor of 3 , consistent with the data reported in Ref. 36. The spectral characteristics of some simulated peaks are superimposed in black in Fig. 5(e). They agree well with the measurements in the whole 
(a)
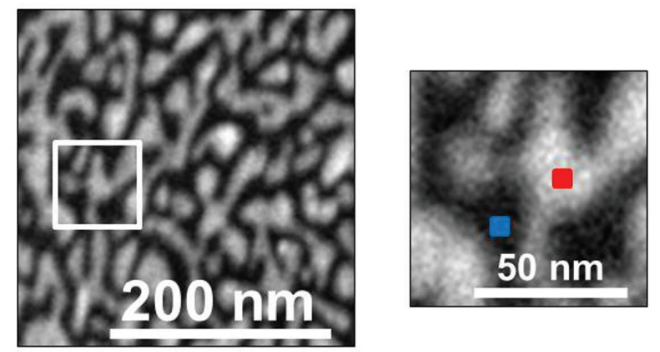

(c)
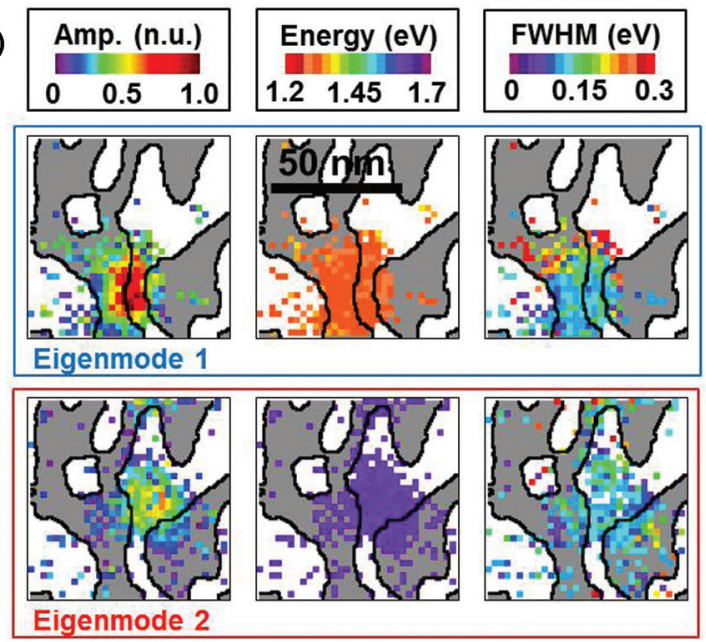

(b)

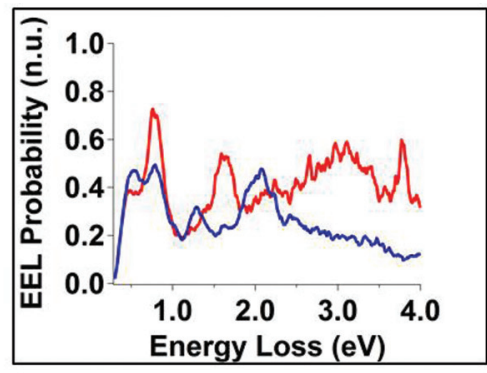

(d)

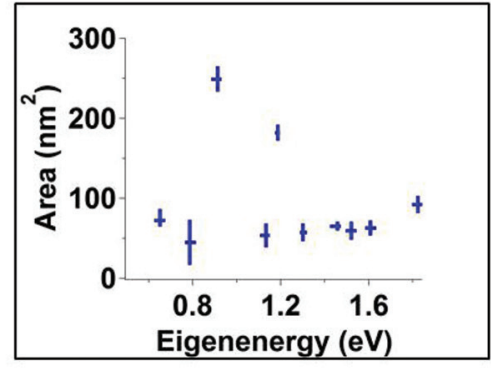

(e)

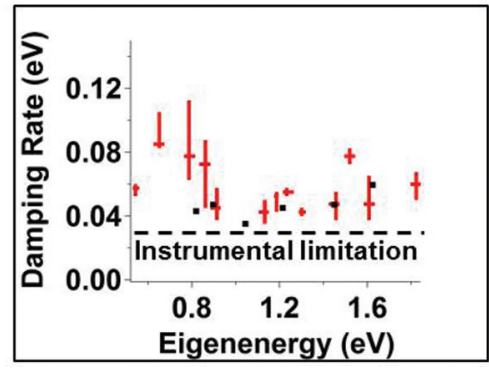

FIG. 5. (Color online) (a) HAADF images of a semicontinuous area and of a small structural detail indicated by the white square. (b) EEL probability local spectra corresponding to the probe positions indicated in (a). (c) Maps of the amplitude (left), central energy (middle), and FWHM (right) of the peaks whose central energy is found within two $0.05 \mathrm{eV}$ wide energy windows centered at 1.30 (top) and $1.60 \mathrm{eV}$ (bottom). When no peak is found in these spectral ranges, the pixel is either blanked if the local material is silver, or greyed if the local material is silicon nitride. (d) Spatial extension of some eigenmodes measured in the semicontinuous area of (a) as a function of their eigenenergy. (e) Red: damping rate of some eigenmodes measured in the semicontinuous area of (a) as a function of their eigenenergy. The black dotted line shows the instrumental limitation imposed by the spectral resolution of the measurement. Black: Half FWHM as a function of the central energy of the EMLDOS peaks obtained after numerical simulation performed in a similar area.

spectral range. The sensitivity of the simulated FWHM to the amplitude of the imaginary part of the dielectric constant seems to indicate that the damping rates of the eigenmodes are dominated by ohmic losses.

\section{CONCLUSION}

In conclusion, we have observed local maxima in the spatial variations of the STEM-EELS signal from semicontinuous films. We found that their strength, energy dependence, and position with respect to the substrate geometry become singular at low energies and around the percolation threshold, yet this peculiarity fades at higher energies. We have been able to extract the complete characteristics of each eigenmode of the systems. From this analysis, we have proven that the medium becomes spatially and spectrally dense in the IR when the fractal geometry of the metallic clusters becomes prominent and confirm early theories on eigenmodes in random films. As another strong difference with eigenmodes of simple systems, we have not observed any obvious correlation between the local morphology of the films and the eigenmodes properties.

These findings, however, let open important questions concerning the physics of these eigenmodes. Indeed, the radiative or nonradiative nature of the modes ${ }^{27}$ is still experimentally unknown. A combination of EELS and high throughput cathodoluminescence experiments ${ }^{37,38}$ could potentially address this issue in the future. Also, the spatial coherence of the modes, which has been heavily debated in the pioneering works, is still experimentally unclear. ${ }^{28}$ Recent developments in electron holography combined with EELS may, however, bring an answer. ${ }^{39}$

\section{ACKNOWLEDGMENTS}

A.L., O.S., and M.K. acknowledge financial support from the French General Directorate for Armament, the National Research Agency under contracts HYNNA and TEMPOS, and the METSA network. The research leading to these results has also received funding from the European Union Seventh Framework Programme under Grant Agreement 312483 ESTEEM2 (Integrated Infrastructure Initiative I3). We thank R. Carminati, G. Boudarham, M. Tencé, and Z. Mahfoud for enjoyable discussions and/or help in experiments or data processing. G.A.B. is grateful to NSERC for a Discovery Grant supporting part of this work. 
*kociak@1ps.u-psud.fr

${ }^{1}$ E. Akkermans and G. Montambaux, Mesoscopic Physics of Electrons and Photons (Cambridge University Press, Cambridge, 2007).

${ }^{2}$ V. A. Markel, L. S. Muratov, M. I. Stockman, and T. F. George, Phys. Rev. B 43, 8183 (1991).

${ }^{3}$ V. M. Shalaev, Phys. Rep. 272, 61 (1996).

${ }^{4}$ M. I. Stockman, V. M. Shalaev, M. Moskovits, R. Botet, and T. F. George, Phys. Rev. B 46, 2821 (1992).

${ }^{5}$ A. K. Sarychev, V. A. Shubin, and V. M. Shalaev, Phys. Rev. B 60, 16389 (1999).

${ }^{6}$ M. Stockman, S. Faleev, and D. Bergman, Phys. Rev. Lett. 87 (2001).

${ }^{7}$ R. F. Voss, R. B. Laibowitz, and E. I. Alessandrini, Phys. Rev. Lett. 49, 1441 (1982).

${ }^{8}$ P. Gadenne, Y. Yagil, and G. Deutscher, J. Appl. Phys. 66, 3019 (1989).

${ }^{9}$ V. M. Shalaev and A. K. Sarychev, Phys. Rev. B 57, 13265 (1998).

${ }^{10}$ L. Novotny, Phys. Rev. Lett. 98, 266802 (2007).

${ }^{11}$ S. Grésillon, L. Aigouy, A. C. Boccara, J. C. Rivoal, X. Quélin, C. Desmarest, P. Gadenne, V. A. Shubin, A. K. Sarychev, and V. M. Shalaev, Phys. Rev. Lett. 82, 4520 (1999).

${ }^{12} \mathrm{~S}$. Ducourtieux, V. Podolskiy, S. Grésillon, S. Buil, B. Berini, P. Gadenne, A. Boccara, J. Rivoal, W. Bragg, K. Banerjee et al., Phys. Rev. B 64, 165403 (2001).

${ }^{13}$ K. Seal, M. A. Nelson, Z. Charles Ying, D. A. Genov, A. K. Sarychev, and V. M. Shalaev, Phys. Rev. B 67, 035318 (2003).

${ }^{14}$ S. Buil, J. Aubineau, J. Laverdant, and X. Quélin, J. Appl. Phys. 100, 063530 (2006).

${ }^{15}$ C. Awada, G. Barbillon, F. Charra, L. Douillard, and J.-J. Greffet, Phys. Rev. B 85, 045438 (2012).

${ }^{16}$ V. Krachmalnicoff, E. Castanié, Y. De Wilde, and R. Carminati, Phys. Rev. Lett. 105, 183901 (2010).

${ }^{17}$ A. Kubo, K. Onda, H. Petek, Z. Sun, Y. S. Jung, and H. K. Kim, Nano Lett. 5, 1123 (2005).

${ }^{18}$ J. Nelayah, M. Kociak, O. Stéphan, F. J. Garcia de Abajo, M. Tencé, L. Henrard, D. Taverna, I. Pastoriza-Santos, L. M. Liz-Marzan, and C. Colliex, Nat. Phys. 3, 348 (2007).

${ }^{19}$ D. Rossouw and G. A. Botton, Phys. Rev. Lett. 110, 066801 (2013).
${ }^{20}$ S. Mazzucco, O. Stéphan, C. Colliex, I. Pastoriza-Santos, L. Liz-Marzan, J. Garcia de Abajo, and M. Kociak, Eur. Phys. J. Appl. Phys. 54, 33512 (2011).

${ }^{21}$ M. Bosman, G. R. Anstis, V. J. Keast, J. D. Clarke, and M. B. Cortie, ACS Nano 6, 319 (2012).

${ }^{22}$ A. Garcia-Etxarri, I. Romero, F. Javier Garcia de Abajo, R. Hillenbrand, and J. Aizpurua, Phys. Rev. B 79, 125439 (2009).

${ }^{23}$ K. Joulain, R. Carminati, J.-P. Mulet, and J.-J. Greffet, Phys. Rev. B 68, 245405 (2003).

${ }^{24}$ F. J. Garcia de Abajo and M. Kociak, Phys. Rev. Lett. 100, 106804 (2008).

${ }^{25}$ G. Boudarham and M. Kociak, Phys. Rev. B 85, 245447 (2012).

${ }^{26}$ E. Castanié, V. Krachmalnicoff, A. Cazé, R. Pierrat, Y. De Wilde, and R. Carminati, Opt. Lett. 37, 3006 (2012).

${ }^{27}$ A. Cazé, R. Pierrat, and R. Carminati, Photon. Nanostructur. 10, 339 (2012).

${ }^{28}$ A. Cazé, R. Pierrat, and R. Carminati, Phys. Rev. Lett. 110, 063903 (2013).

${ }^{29}$ S. Camelio, D. Babonneau, D. Lantiat, L. Simonot, and F. Pailloux, Phys. Rev. B 80, 155434 (2009).

${ }^{30}$ A. Gloter, A. Douiri, and M. Tencé, and C. Colliex, Ultramicroscopy 96, 385 (2003).

${ }^{31}$ M. Bosman, E. Ye, S. F. Tan, C. A. Nijhuis, J. K. W. Yang, R. Marty, A. Mlayah, A. Arbouet, C. Girard, and M.-Y. Han, Sci. Rep. 3, 1312 (2013).

${ }^{32}$ A. Bossavit, IEEE Trans. Magn. 24, 74 (1988).

${ }^{33}$ Y. Yagil, M. Yosefin, D. J. Bergman, G. Deutscher, and P. Gadenne, Phys. Rev. B 43, 11342 (1991).

${ }^{34}$ M. I. Stockman, L. N. Pandey, and T. F. George, Phys. Rev. B 53, 2183 (1996).

${ }^{35}$ P. B. Johnson and R. W. Christy, Phys. Rev. B 6, 4370 (1972).

${ }^{36}$ M.-L. Thèye, Phys. Rev. B 2, 3060 (1970).

${ }^{37}$ N. Yamamoto, K. Araya, and F. J. G. de Abajo, Phys. Rev. B 64, 205419 (2001)

${ }^{38}$ L. F. Zagonel, S. Mazzucco, M. Tencé, K. March, R. Bernard, B. Laslier, G. Jacopin, M. Tchernycheva, L. Rigutti, F. H. Julien et al., Nano Lett. 11, 568 (2011).

${ }^{39}$ H. Lichte, Microscopy 62, S17 (2013). 